\title{
Enseñanza de la Prehistoria en primaria: análisis de los esquemas mentales sobre el origen del ser humano en estudiantes de sexto curso $^{1}$
}

\author{
Teaching of Prehistory in primary: analysis of \\ the mental schemas on the origin of the hu- \\ man being in sixth-year students
}

\author{
Cristhian Abate Flores* \\ abaflo08@gmail.com
}

\section{Resumen}

La Teoría de la Evolución es considerada como una revolución en el pensamiento científico, así como uno de los pilares para el entendimiento de la ciencia moderna, por ello, la importancia y el impacto que los conocimientos derivados de esta teoría pueden tener en la sociedad como un eje fundamental en el estudio de cualquier incursión académica, por ende, en el presente estudio, se analizan los conceptos nucleares de 161 estudiantes de sexto curso de la ciudad de Badajoz, sobre el origen del ser humano en la Prehistoria, utilizando test de tipo mapa cognitivo, se aprecian tres esquemas: creacionista evolucionista - mixto, y la predilección de los estudiantes por los modelos de carácter religioso ante los científicos.

Este estudio es parte de los resultados del trabajo de fin de Máster: “La enseñanza de la Prehistoria en la educación primaria: análisis de contenido, de libros de texto y estructuras mentales en relación con el origen del ser humano" para la obtención del Máster en Investigación de la Enseñanza y Aprendizaje de las Ciencias Experimentales, Sociales y Matemáticas, Universidad de Extremadura, España.

* Investigador Independiente/ Estudiante postgrado Universidad de Extremadura, España Recibido 11 de agosto 2017 / Aceptado 20 de octubre 2017. 
Palabras Clave: Teoría de la Evolución, Creacionismo, Diseño Inteligente, Conceptos Nucleares.

\begin{abstract}
The Theory of Evolution is considered as a revolution in scientific thinking, as well as one of the pillars for the understanding of modern science; thus, the importance and the impact that the knowledge derived from this theory can have in society are fundamental axis in the educational systems. Therefore, in the present study, the

nuclear concepts of 161 students of the sixth year of the city of Badajoz are analyzed about the origin of the human being in Prehistory, using a cognitive map type test, three schemes were observed: creationist - evolutionist - mixed, and the students' predilection for models of religious character before scientists.
\end{abstract}

Keywords: Theory of Evolution, Creationism, Intelligent Design, Nuclear Concepts.

\title{
Introducción
}

La educación primaria es parte fundamental en el desarrollo intelectual y personal del individuo, ya que forma parte de lo que algunos autores (Van Lieshout, 1986, pp. 122-124) Ilaman "hechos vitales normativos" de carácter social o cognitivo, estos implican tareas de desarrollo y formación, las cuales constituyen la submuestra de todas las labores que una persona plena deba dominar a lo largo de su vida, es decir, la formación integral del sujeto.

A demás de ello, la escuela primaria induce al sujeto en los procesos de enseñanza, donde convergen las historias y experiencias de cada uno, así como el contenido de carácter científico y sistemático, dichos contenidos, tienen una complejidad propia dentro de la escuela 
Enseñanza de la Prehistoria en primaria: análisis de los esquemas mentales sobre el origen del ser humano en estudiantes de sexto curso

primaria, ya que entra en juego un tridente de carácter conceptual: 1) El "saber genético puro" concebido de las ramas científicas. 2) El "saber digerido" y transmitido por el maestro.3) Lo que percibe el estudiante. Por esta gran complejidad científica y por la iniciación de saberes formales se justifica el estudio de algunos contenidos en primaria que requieren de mucha atención (Shubauer-Leoni, 1986, pp. 103-106).

Dentro de esos contenidos complejos y polifacéticos, se encuentra La Teoría de la Evolución, la cual, además de esa peculiaridad de complejidad es fundamental en el estudio de la ciencia moderna. Al respecto Fernández y San José (2007) afirman que "Comprender el mecanismo evolutivo permite acceder a otros conceptos tales como las taxonomías, los ecosistemas o el flujo de materia y energía en los mismos. Además, el disponer de un marco conceptual correcto sobre la Evolución y la herencia genética, permite a los alumnos entender los últimos avances en el campo de la biotecnología" (p. 130). En este sentido, cabe agregar no solamente el área de las Ciencias Experimentales, también podría decirse que el comprender esta teoría, ayuda a concebir conceptos de áreas de las Ciencias Sociales, como los procesos de humanización, evolución del hombre y mujer modernos, y la cultura que hoy conocemos.

Cabe resaltar, que en paralelo a la importancia que implica comprender dicho conjunto de saberes, radica la complejidad que requiere su enseñanza y aprendizaje, esto demostrado en varios trabajos realizados desde la línea de las ideas alternativas en la escuela secundaria (Carrascosa, Furió y Solbes, 2006, pp. 66-68, ver también, Jiménez, 1989,2004; Fernández y San José 2007) que colocan al estudio de la Teoría de la Evolución y sus derivaciones como una idea alternativa de núcleo fuerte; otros trabajos realizados desde el análisis de los libros escolares relacionados a la Teoría de la Evolución demuestran la presencia de conceptos erróneos que fortalecen el núcleo de las ideas alternativas relacionadas a este tema y por ende la obstaculización en la enseñanza de la ciencia en la escuela ( De la Gandara, Gil\& San Matín, 2002, pp. 311-313, ver también, De la Gandara, 1999).

<Paradigma> - Revista de Investigación Educativa. Año 24. No. 38 
Las pretensiones de este estudio, en relación con lo que se ha planteado en párrafos anteriores, es un acercamiento al estado del concepto sobre el origen del ser humano en primaria, partiendo de la aplicación de instrumentos de tipo mapa cognitivo a 165 estudiantes de sexto grado en 7 diferentes escuelas, esto para analizar sus esquemas mentales en torno al tópico de interés, para ello se emplearon los supuestos de la Teoría de Núcleos Conceptuales (en adelante TCN).

\section{Métodos y Materiales}

Este estudio corresponde a una sección del trabajo de fin de Máster sobre el tratamiento del origen del ser humano en primaria, dicha fase es cuantitativa, con un diseño descriptivo y con una muestra por conveniencia. En esta unidad se decidió la construcción de estructuras cognitivas utilizando como modelo el tipo de organización de los mapas cognitivos de "pregunta de enfoque y estancamiento" los cuales consisten en que el estudiante parta de una pregunta "foco" para la elaboración de su esquema mental, mientras que el segundo consiste en proporcionarle al estudiante una lista de conceptos para la elaboración de un esquema² (Novack y Cañas, 2006, sección de Parking Lot \& Expert Skeleton Maps).

En el Procesamiento de los datos se utilizó solamente el modelo de representación de los esquemas mentales de Novack ${ }^{3}$, donde no se estimó la jerarquía de los conceptos, sino el recuento de las relaciones entre un concepto con otro, así, para la producción de los núcleos conceptuales, se realizó un recuento de las combinaciones usadas por esquema en relación con el total de combinaciones posibles de cada modelo (creacionista / evolucionista 21 combinaciones posibles, modelo mixto 59), aplicada en matrices binarias ${ }^{4}$, luego de ello, se eliminaron las frecuencias de uso débiles y se utilizaron las de

\footnotetext{
2 Se puede apreciar un ejemplo del mapa cognitivo en la figura 2.

3 A diferencia de la Teoría de Mapas conceptuales, donde el conocimiento se ordena en categorías jerárquicas, aquí se considera la proximidad entre un concepto con otro, creando núcleos y periferias.

4 Se pueden observar algunos ejemplos de las combinaciones entre pares en las figuras 5 y 6 .
} 
Enseñanza de la Prehistoria en primaria: análisis de los esquemas mentales sobre el origen del ser humano en estudiantes de sexto curso

porcentaje fuerte ( $>20 \%$ / 30\%) para la construcción de los modelos mentales representados con el programa Gephi, donde los nodos representan conceptos y el grosor de las aristas, el porcentaje de las frecuencias.

\section{Discusión teórica}

$\bullet \bullet \bullet \bullet \bullet \bullet \bullet \bullet \bullet \bullet \bullet \bullet \bullet \bullet \bullet \bullet \bullet \bullet \bullet \bullet \bullet \bullet \bullet \bullet \bullet \bullet \bullet \bullet \bullet \bullet \bullet \bullet \bullet \bullet \bullet \bullet \bullet \bullet \bullet \bullet \bullet \bullet$

Representación de Redes Cognitivas: Teoría de Núcleos Conceptuales

La TCN, surge como una alternativa hacia algunas teorías tradicionales sobre la estructuración cognitiva y los esquemas de aprendizaje, principalmente aquellas que suponen una organización jerárquica y con tendencias a la complejidad a medida se desarrollen procesos de aprendizaje y adquisición de un mayor número de conceptos (Luengo, R., Casas, L., Masas, J., \& Contreras J., 2014, sección de Introducción y de Fundamentos: la Teoría de los Conceptos Nucleares).

La TCN tiene como base, la estructuración del conocimiento a partir de metáforas relacionadas al posicionamiento geográfico, argumentadas bajo los supuestos extraídos desde las ciencias dedicadas a la educación y a los procesos cognitivos(Casas, Luengo, Canchado, \& Torres, 2013, pp.2-3); la TCN abarca tres ejes fundamentales: 1) El conocimiento geográfico, 2) Los conceptos nucleares y 3) Las rutas de mínimo costo.

Dentro del conocimiento geográfico sobresalen tres etapas: a la primera de estas, se le conoce como "adquisición de hitos" ligado a la asociación de un nuevo concepto con las ideas previas o referencias cotidianas. A estos conceptos previos dentro de la TCN se les conoce como "hitos", mientras que al conjunto de todos los conceptos - nuevos y previos - se les conoce como "las redes conceptuales". Por su parte, la segunda fase es conocida como "el conocimiento de rutas" las cuales se entienden como las relaciones que hay entre todos los "hitos" (conceptos nuevos y previos), por último, "el conocimiento de conjunto" donde se integran todos los conocimientos del entorno, es decir, los conceptos, nuevos y previos, y las relaciones que existen entre ellos,

<Paradigma> - Revista de Investigación Educativa. Año 24. No. 38 
construyendo así un mapa o una estructura cognitiva general en referencia a un fenómeno en particular (Luengo, 2013, pp.17-20).

Otros de los fundamentos de la TCN, es el caso de las rutas de mínimo costo, estas tienen como fundamento experiencias investigativas sobre las redes cognitivas desde un diseño longitudinal realizadas por el grupo Ciberdidact ${ }^{5}$, dichas investigaciones contradicen en gran medida los supuestos de la Ciencia Cognitiva tradicional en los apartados de complejidad reciente, la cual plantea que a medida se posiciona de mayor número de conceptos, las redes cognitivas se hacen más complejas, sin embargo, los resultados de este grupo de investigación arrojan lo contrario, ya que, según estudios desde el área de las matemáticas, a medida el estudiante amplía su bagaje conceptual, su red se va haciendo menos compleja, dicho fenómeno, es explicado según los autores de esta teoría, bajo los parámetros de los supuestos de Eldeman sobre la selección de los grupos neuronales, la cual supone la activación de los circuitos neuronales más eficientes para el ahorro energético, eliminando los circuitos ineficientes, reduciendo la complejidad por la eficiencia; así sucede en las redes cognitivas, a medida el sujeto tiene un mayor panorama conceptual, solamente utiliza aquellos conceptos que le resulten más eficientes para llegar a una respuesta lógica y que satisfaga su necesidad (Luengo, 2013, pp. 20-21).

\section{Teorías sobre el origen del ser humano}

El cuestionamiento a la naturaleza de los seres y por qué existen las formas de vidas que hoy día conocemos, ha generado conflictos intelectuales a lo largo de la historia, así se podría decir que existen varias concepciones en cuanto al origen de la especie humana y de la vida en general; una basada en hechos científicos, otra en creencias religiosas y una concepción hibrida donde convergen los hechos científicos sin dejar fuera el sentir devoto, es por ello que los términos evolución y creación se tornan multifacéticos y convergen en algunos casos.

5 Este es el grupo de Investigación de la Universidad de Extremadura dedicada al estudio de la TCN. 
Enseñanza de la Prehistoria en primaria: análisis de los esquemas mentales sobre el origen del ser humano en estudiantes de sexto curso

Como ejemplo de lo anterior podemos decir que por un lado están los que creen en la evolución como un plan divino, un sentido teleológico, que creen que los cambios suceden porque alguien así lo diseñó (diseño Inteligente), también están los que piensan que los datos bíblicos son fenómenos literales, como la formación de la tierra en siete días o la creación de Adán y Eva por medio del barro (creacionistas), y los que solamente se basan en hechos científicos apoyados en los avances y aportaciones de ciencias como la Genética, Biología, Paleontología, Geología, etc. (Warnick, 2014, p. 56).

Las concepciones científicas tienen como fundamento elementos de las teorías de la evolución y de la genética moderna, convirtiéndose en lo que hoy día se conoce como Síntesis Moderna (Ministerio de Educación de la Nación, 2012). La evolución como proceso observable en la naturaleza se puede resumir en palabras de Corredor (2007) con las siguientes características:

...que las especies producen más progenie de la que realmente puede sobrevivir y reproducirse, que esa capacidad de los organismos de sobrevivir y reproducirse es variable (que es lo que hoy en día conocemos como adaptabilidad o fitness diferencial) y finalmente, que la variación es heredable (p.37).

Por ende, el evolucionismo tradicional surge de dos líneas enmarcadas en las leyes biológicas, observables y experimentales de la sexualidad y en la adaptación al medio de las especies, esto en yuxtaposición con las demás posturas basadas en la inmutabilidad de las especies de una generación a otra.

<Paradigma> - Revista de Investigación Educativa. Año 24. No. 38 


\section{Resultados}

Figura 1

Resultados de los esquemas mentales de los estudiantes de sexto grado en relacion al origen del ser humano

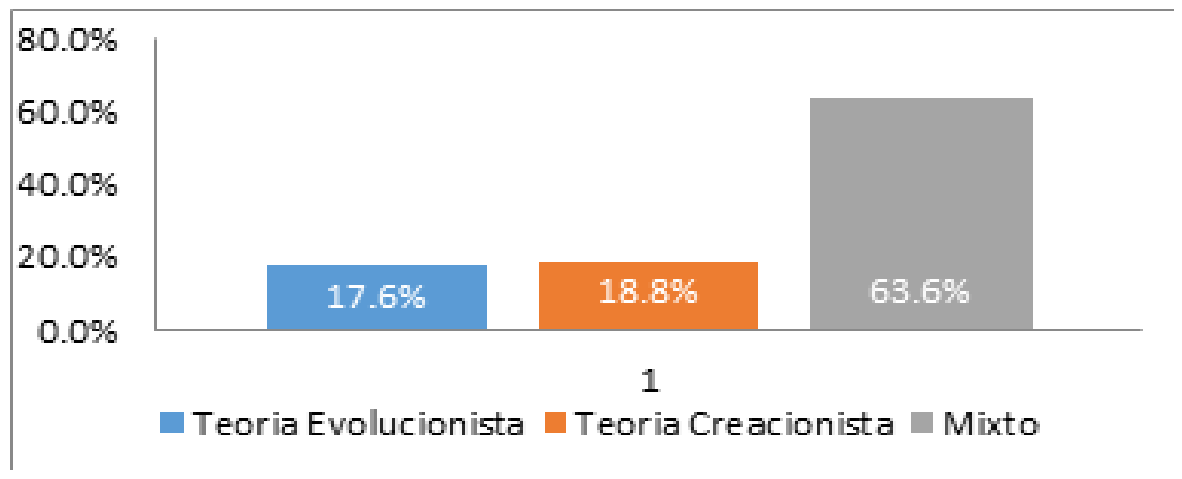

Tabla 1

Porcentajes de los esquemas mentales de niños de sexto de primaria en relacion al origen del ser humano

\begin{tabular}{cccccc}
\hline \multicolumn{5}{c}{ Esquema } \\
\hline \multicolumn{7}{c}{ Teoría de la Evolución } & \multicolumn{2}{c}{ Creacionismo } & \multicolumn{2}{c}{ Diseño Mixto } \\
\hline Recuento & \% de la fila & Recuento & \% de la fila & Recuento & \% de la fila \\
\hline 29 & $17.6 \%$ & 31 & $18.8 \%$ & 101 & $63.6 \%$ \\
\hline
\end{tabular}

En la aplicación del test al total de la muestra (161 estudiantes) emergieron tres esquemas o modelos mentales sobre la concepcion del origen del ser humano ${ }^{6}$, dichos esquemas se agruparon en funcion a la selección de palabras por parte de los estudiantes relacionadas a las dos categorías establecidas (creacionista - evolucionista), asicon la selección de palabras para la construccion del esquema mental, los

\footnotetext{
6 En las figuras 2, 5 y 6 se pueden observar ejemplos de los esquemas realizados por los estudiantes, donde se aprecian los tres tipos de modelos en función de la selección y combinación de palabras de cada categoría.
} 
Enseñanza de la Prehistoria en primaria: análisis de los esquemas mentales sobre el origen del ser humano en estudiantes de sexto curso

niños que combinaron conceptos creacionistas con evolucionistas representan el $60.84 \%$, es decir, el esquema mixto, mientras los que solo usaron palabras creacionista son el $21.08 \%$, el esquema con menos porcentaje es el evolucionista (18.07\%).

\section{Esquema de las estructuras creacionistas}

Para la elaboración del análisis de los Núcleos Conceptuales, se tomó en cuenta las relaciones existentes entre los siete conceptos que componen el listado creacionista (cuatro conceptos religiosos y tres neutrales), así, mediante el porcentaje de frecuencia de las combinaciones entre pares, se realizó un corte en el $20 \%$, eliminando las relaciones menores a este porcentaje por considerarse débiles; la matriz binaria que sirvió para la obtención de los conceptos que conforman los núcleos conceptuales es la siguiente:

Tabla 2

Relaciones binarias entre los conceptos del esquema creacionista

\begin{tabular}{|c|c|c|c|c|c|c|c|}
\hline & N1 & N2 & N3 & C1 & C2 & C3 & C4 \\
\hline N1 & & $0 \%$ & $3.2 \%$ & $61.3 \%$ & $9.7 \%$ & $0 \%$ & $12.9 \%$ \\
\hline N2 & & & $3.2 \%$ & $3.2 \%$ & $6.5 \%$ & $3.2 \%$ & $3.2 \%$ \\
\hline N3 & & & & $3.2 \%$ & $22.6 \%$ & $9.7 \%$ & $12.9 \%$ \\
\hline C1 & & & & & $83.9 \%$ & $6.5 \%$ & $12.9 \%$ \\
\hline $\mathrm{C2}$ & & & & & & $25.8 \%$ & $64.5 \%$ \\
\hline C3 & & & & & & & $45.2 \%$ \\
\hline
\end{tabular}

Nota: Factor de ponderacion $>\mathbf{2 0} \%$ en Negrita. N1=Hace millones de años; N2= Hace miles de años; N3 = Animales; $\mathrm{C} 1$ = Dios; $\mathrm{C2}=$ Creacion; $\mathrm{C} 3$ = Paraiso; $\mathrm{C} 4=$ Adan y Eva. 


\section{Figura 2}

Ejemplo de las seis relaciones del esquema de las estrcuturas creacionistas realizado por uno de los informantes

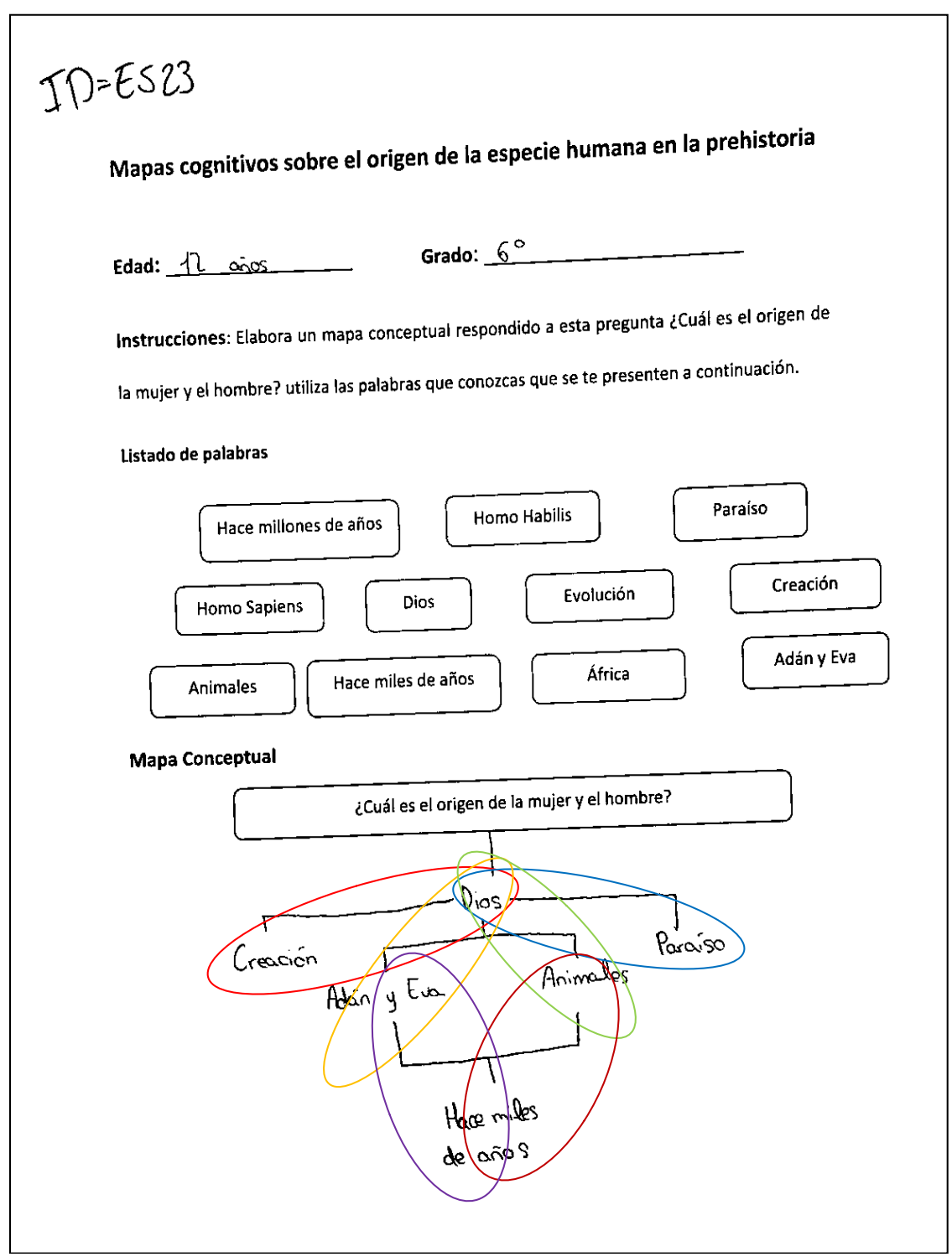

Nota: Las relaciones son: Dios - Creación $=$ rojo $/$ Dios - Paraíso $=$ azul $/$ Dios - Adán y Eva $=$ amarillo $/$ Dios - Animales $=$ verde $/$ Adán y Eva - Hace miles de años $=$ morado / Animales - Hace miles de años = ocre 
Enseñanza de la Prehistoria en primaria: análisis de los esquemas mentales sobre el origen del ser humano en estudiantes de sexto curso

Los resultados sobre los conceptos nucleares y polares se presentan en la siguiente figura:

Figura 3

Estructura de los conceptos nucleares del esquema mental creacionista

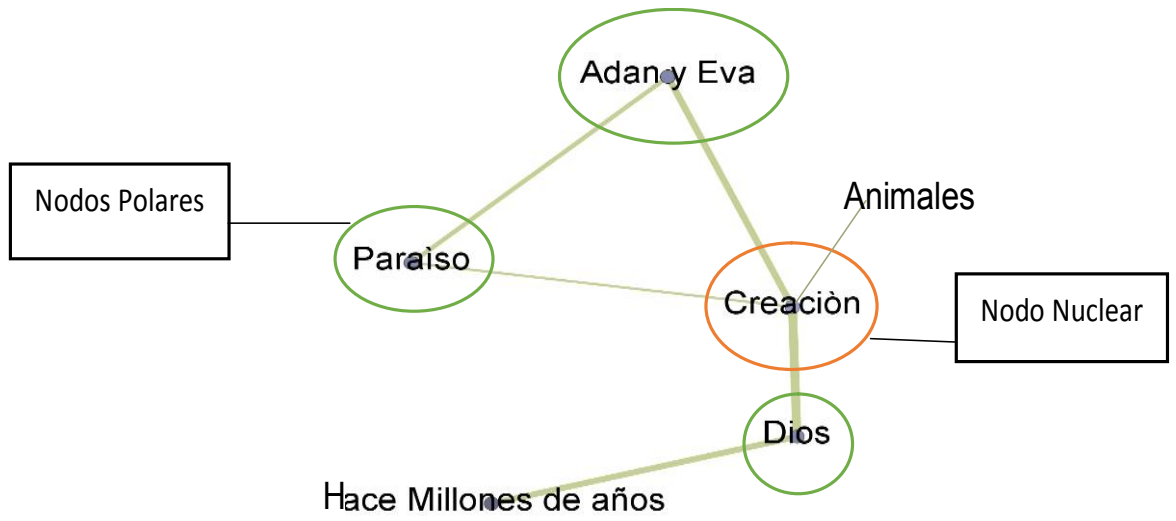

Nota: En la representacion de tipo red cognitiva, los nodos representan los conceptos utilizados y el grosor de las aristas es correlativo al numero de relaciones dadas en los esquemas de los estudiantes.

Dentro del esquema presentado en la figura 3, se aprecia que el modelo mental de los estudiantes con un esquema creacionista es compuesto por seis conceptos, de los cuales, tres representan nodos polares (relación con dos conceptos): el concepto de Dios, el de Adán y Eva y Paraíso; el nodo nuclear (relación con más de dos conceptos) y alrededor del cual giran los demás nodos, es el concepto de creación, este se relaciona con Paraíso, Adán y Eva, y Dios.

\section{Esquema de las estructuras evolucionistas}

En el esquema evolucionista se realizó un corte en el 30\% a diferencia del esquema creacionista donde se decidió hacer en el $20 \%$, eliminado las relaciones menores a este porcentaje por considerarse débiles; la matriz binaria es la siguiente:

<Paradigma> - Revista de Investigación Educativa. Año 24. No. 38 
Tabla 3

Relaciones binarias entre los conceptos del esquema evolucionista

\begin{tabular}{|c|c|c|c|c|c|c|c|}
\hline & N1 & N2 & N3 & E1 & E2 & E3 & E4 \\
\hline N1 & & $3.4 \%$ & $41.4 \%$ & $24.1 \%$ & $10.3 \%$ & $31.0 \%$ & $6.8 \%$ \\
\hline N2 & & & $0.0 \%$ & $3.4 \%$ & $6.8 \%$ & $6.8 \%$ & $3.4 \%$ \\
\hline N3 & & & & $41.1 \%$ & $13.8 \%$ & $6.8 \%$ & $6.8 \%$ \\
\hline E1 & & & & & $38.0 \%$ & $6.5 \%$ & $3.4 \%$ \\
\hline E2 & & & & & & $58.6 \%$ & $3.4 \%$ \\
\hline E3 & & & & & & $27.6 \%$ & $3.4 \%$ \\
\hline E4 & & & & & & & \\
\hline
\end{tabular}

Nota: Factor de ponderacion $>20 \%$ en Negrita. N1=Hace millones de años; N2= Hace miles de años; N3 = Animales; E1 = Evolucion; E2 = Homo Habilis; E3 = Homo Sapiens; $\mathrm{E} 4$ = Africa.

\section{Figura 4}

Ejemplo de una estructura mental de carácter creacionista de uno de los informantes

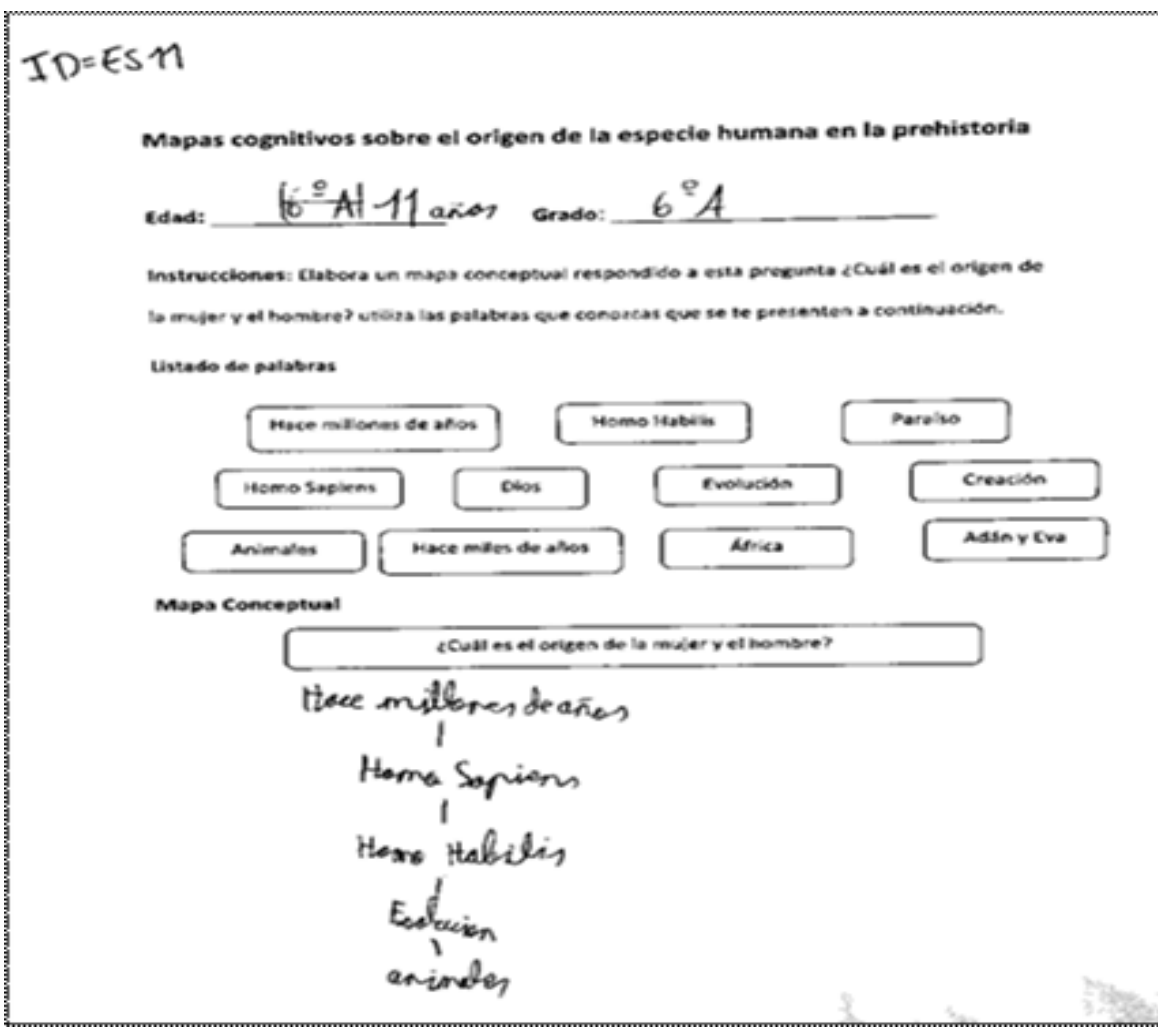

64 
Enseñanza de la Prehistoria en primaria: análisis de los esquemas mentales sobre el origen del ser humano en estudiantes de sexto curso

Los resultados sobre los conceptos nucleares y polares se presentan en la siguiente figura:

Figura 5

Estructura de los conceptos nucleares del esquema mental evolucionista

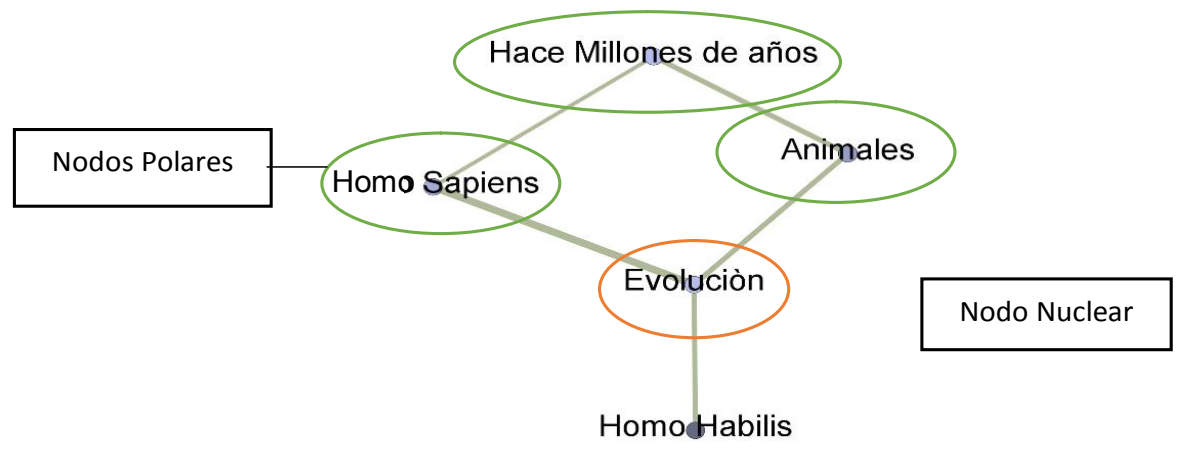

En esta figura 5 podemos apreciar que el concepto en el que gira el esquema, es el de evolución, el cual es nuestro concepto nuclear y se relaciona con los conceptos de Homo Sapiens, animales y Homo Habilis; los conceptos polares son: Homo Sapiens, animales y hace millones de años.

\section{Esquemas de las estructuras mixtas}

Para la elaboración del esquema mixto, se tomó en cuenta las relaciones existentes entre todos los conceptos (11 en total y 59 posibles combinaciones) y elaborando el corte entre las relaciones fuertes y débiles en el $20 \%$; la matriz binaria que sirvió para la obtención de los conceptos que conforman los núcleos conceptuales es la siguiente:

<Paradigma - Revista de Investigación Educativa. Año 24. No. 38 


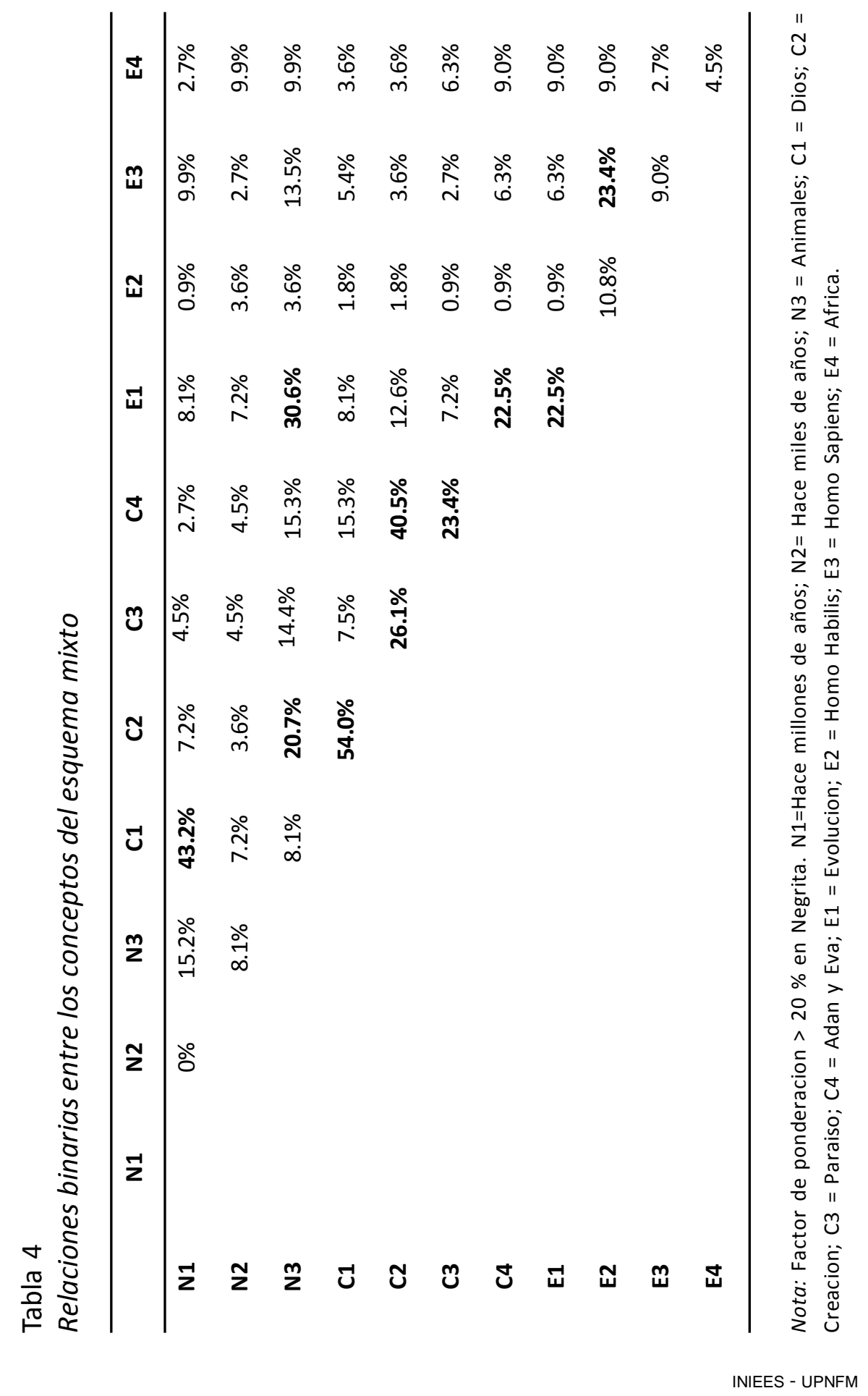

66 
Enseñanza de la Prehistoria en primaria: análisis de los esquemas mentales sobre el origen del ser humano en estudiantes de sexto curso

Figura 6

Ejemplo de una estructura mental de carácter mixto

de uno de los informantes

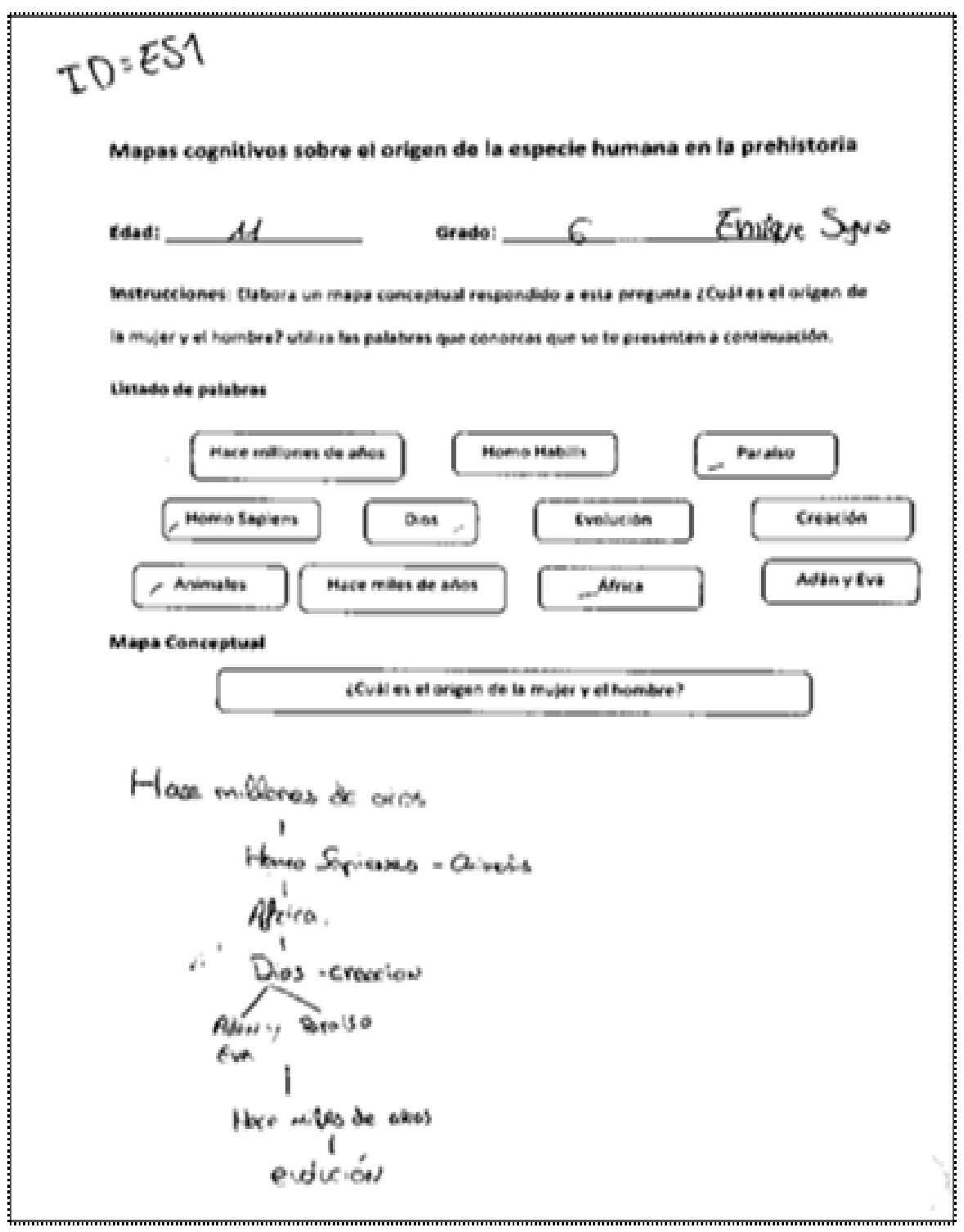

El esquema elaborado a partir de la selección de dichas relaciones se muestra en la siguiente figura:

<Paradigma> - Revista de Investigación Educativa. Año 24. No. 38 
Figura 7

Estructura de los conceptos nucleares del esquema mental Mixto

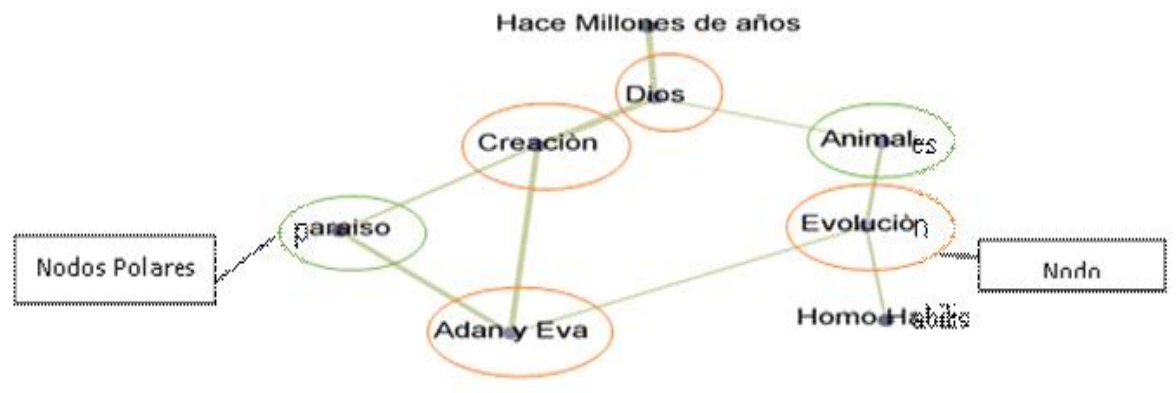

En este esquema, se aprecia que la estructura mental de los niños con un tipo de pensamiento mixto respecto a nuestro tópico de interés, tienen cuatro conceptos nucleares que sirven como ejes de esquema, estos son: Adán y Eva, Evolución, Dios y Creación, cada uno de ellos relacionados a tres conceptos, mientras que los esquemas polares son dos, Paraíso y Animales, relacionados a dos conceptos cada uno.

\section{Conclusiones}

En los esquemas mentales se aprecia de manera contundente que los estudiantes que fueron parte de esta muestra tienen tres modelos asociados a la idea del origen del ser humano, dichos esquemas son: el creacionista, basado en los relatos bíblicos, el evolucionista, argumentado mediante pruebas científicas en el proceso evolutivo de las especies antecesores al humano y el mixto, el cual se construye en función de argumentos científicos y religiosos. De estos esquemas, la mayoría de estudiantes tiene un modelo mental mixto $(63,6 \%)$, a este le siguen el esquema creacionista (18.6\%) y por último el evolucionista (17.8\%), dichos esquemas están acorde a lo que muchos teóricos llegan a concluir en las percepciones acerca del origen de la vida: Teoría Evolucionista, Creacionismo Tradicional y Diseño Inteligente (Warnick, 2014, p.56). 
Enseñanza de la Prehistoria en primaria: análisis de los esquemas mentales sobre el origen del ser humano en estudiantes de sexto curso

Dentro del esquema mixto, los estudiantes asocian una serie de conceptos al origen del ser humano, dentro de los cuales existe un núcleo conceptual estructurado de cuatro conceptos: Dios, Creación, Evolución, y Adán y Eva, dando a conocer una marcada estima de los conceptos religiosos sobre los científicos, ya que básicamente responde al proceso figurado en los relatos bíblicos, con la alteración que supone la incorporación del concepto de evolución, mientras que el núcleo polar lo componen los conceptos de Paraíso y Animales, así se entiende, que estos piensan que el origen del ser humano proviene de Dios, la creación, la evolución y Adán y Eva. Dichas ideas, quedan expuestas bajo los supuestos del Diseño Inteligente, donde existe una combinación entre los argumentos científicos y los religiosos, dándole una interpretación no literal a los relatos bíblicos, siempre una concepción religiosa (Betancur, 1999, pp. 229-232).

En el esquema creacionista, la concepción acerca del origen del ser humano está asociada fuertemente al concepto de creación, ya que este es el concepto nuclear asociada a otros conceptos polares como Dios, Paraíso, Animales, y Adán y Eva; dicho esquema tiene mucha coherencia con el relato bíblico, dado la naturaleza de la pregunta que se le hizo a los estudiantes, ya que en ambos casos, la respuesta comprende la misma lógica, donde la aparición de los primeros humanos, surge a través de la creación (Génesis 1:1-3,24-27).

Los dos esquemas, el mixto y el de la creación suponen casi el $80 \%$ de la población total del estudio. Estos tienen una concepción relacionada con los relatos bíblicos, ello, en coherencia con el tratamiento que se le da a este tópico dentro de la educación primaria, ya que, tanto en el currículo como en los libros de texto, existe mayor presencia de contenido ligado a la Religión Católica que a las Ciencias Sociales o las Ciencias Naturales (Abate \&Montaña, 2017, pp. 53-57).

En el esquema evolucionista, la idea del origen del ser humano está ligada a la evolución como concepto nuclear, rodeada de conceptos como Homo Sapiens, animales y Homo Hábiles, teniendo una coherencia con los supuestos científicos que manifiestan los procesos 
evolutivos de los seres vivos mediante la selección natural, adaptación al medio y la herencia genética (Ayala, 2009, pp. 103-111, ver también, Darwin 1859/1962, pp. 52-76).

Al analizar el esquema general de los estudiantes que representan esta muestra, se puede asumir que existe una relación fuerte a los argumentos de corte religioso al referirnos al origen del ser humano, ya que la asociación a dichos conceptos prevalece tanto en el esquema creacionista como en el mixto, por ende, al obtener poco contenido y disposiciones curriculares que la ciencia ofrece con respecto al tema sobre el origen de la especie humana, el vacío académico de esta interrogante es solventado por la vía religiosa, la cual se refleja en las estructuras mentales que tienen los estudiantes al respecto.

\section{Recomendaciones}

En primera instancia, se aclara que las recomendaciones se harán dentro del contexto educativo hondureño, ya que la moción de esta publicación, análoga a la puesta en vista de los resultados, es, incentivar la investigación de los procesos de enseñanza y aprendizaje en los contextos científicos en Honduras en todos los niveles educativos, a lo que la metodología implementada en este estudio puede ser una referencia de bastante provecho.

En el caso español, se plantea una revisión al tratamiento pedagógico en conceptos de esta índole en los nivele inferiores del sistema educativo, ya que, a partir de los resultados de este trabajo y de otros referidos a ideas alternativas de la escuela secundaria, se manifiestan obstáculos que impiden la libre comprensión por parte de los estudiantes de los procesos evolutivos y sus derivaciones, por ende, investigaciones como estas y el replanteamiento al tratamiento de dicho concepto, mejorarían los resultados de comprensión científica. 
Enseñanza de la Prehistoria en primaria: análisis de los esquemas mentales sobre el origen del ser humano en estudiantes de sexto curso

\section{Referencias Bibliográficas}

Abate, C., \& Montaña, J. (2017). Tratamiento del origen del ser humano en Educación Primaria: Análisis del currículo, de libros de texto y estructuras mentales de los estudiantes. (Trabajo de Fin de Master) Universidad de Extremadura, Badajoz. pp. 53-57.

Ayala, F. (2009). Darwin y el diseño inteligente. pp. 103-111. Bilbao: Ediciones mensajero.

Betancur, D. (1999). La controversia creacionismo - evolución. Anuario de filosofía del derecho, 16(1), pp. 229-232. Recuperado de https:// dialnet.unirioja.es/descarga/articulo/142399.pdf

Carrascosa, J. Furió, C. Solbes, J. (2006). Las ideas alternativas sobre conceptos científicos: tres décadas de investigación. Revista Alambique, 48(1) pp 66-68. Recuperado de http://alambique.grao.com/ revistas/alambique/48-viejos-temas-nuevos-enfoques/las-ideasalternativas-sobre-conceptos-cientificos-tres-decadas-deinvestigacion.

Casas, L., Luengo, R., Canchado, M., \& Torres, R. (2013). Una experiencia de representación del conocimiento en Educación Infantil mediante el uso de Redes Asociativas Pathfinder. Revista de Educación a Distancia, 36(1), pp. 2-3. Recuperado de http://revistas.um.es/red/ article/view/233681.

Corredor, V. (2007). Neodarwinismo y la Nueva Síntesis. En Rosa A, Filosofía, Darwinismo y evolución, p. 37. Bogotá: Universidad Nacional de Colombia.

Darwin, C. (2009) On the Origin of Species by Means of Natural Selection, or the Preservation of Favoured Races in the Struggle for Life. En Zulueta, A. (Trad.) El origen de las especies por la selección natural. Los libros de la Catarata. Madrid. pp. 52-76. (Trabajo original publicado en 1859). 
De la Gandar, M. (1999). La transposición didáctica del concepto de adaptación biológica. (Tesis doctoral). Universidad de Zaragoza, Zaragoza.

De la Gandara, M.., Gil, M.. \& Sanmartí, P. (2002). Del modelo científico de adaptación biológica al modelo adaptación biológica de los libros de texto de enseñanza secundaria obligatoria. Enseñanza de la Ciencia, 20(2), pp. 303-314. Recuperado de http://www.raco.cat/index.php/ Ensenanza/article/view/21814

La Biblia: El libro del pueblo de Dios (s. f.) Traducción Argentina. (1990). Genesis 1:1-3, 24-27. Recuperado de http://w2.vatican.va/content/ vatican/es.html.

Fernandez, J., \& Sanjose, V. (2007). Permanencia de ideas alternativas sobre Evolución de las Especies en la población culta no especializada. Didáctica de las Ciencas Experimentales y Sociales, 21(1), p, 130.Recuperado de https://ojs.uv.es/index.php/dces/article/view/2427

Jiménez, A. (1989). Los esquemas conceptuales sobre la Selección Natural: Análisis y propropuestas. (Tesis Doctora). Universidad Complutense de Madrid, Madrid.

Jimenéz, A. (2004). El modelo de evolución de Darwin y Wallace en la enseñanza de la biología. Alambique, pp. 72-81. Recuperado de https:/ / w w w. re se a r ch gat e. net/ p u blic a t i o n / 39211930_El_modelo_de_evolucion_de_Darwin_y_Wallace_en_la_ ensenanza_de_la_Biologia

Luengo, R. (2013). La Teoría de los Conceptos Nucleares y su aplicación en la investigación en la didáctica de las matemáticas. Revista Iberoamericana de educación matemáticas, 34(1), pp. 17-21. Recuperado de http://www.fisem.org/www/union/revistas/2013/34/ archivo5.pdf 
Enseñanza de la Prehistoria en primaria: análisis de los esquemas mentales sobre el origen del ser humano en estudiantes de sexto curso

Luengo, R., Casas, L., Masas, J., \& Contreras J. (Septiembre, 2014). Índices de Nuclearidad (Completo y Reducido), como aportación a la Teoría de Conceptos Nucleares. Revista Ibérica de Sistemas y Tecnologías de Información,4(1), Sección de Introducción y Fundamentos. La teoría de los Conceptos Nucleares, Recuperado de http://www.scielo.mec.pt/scielo.php?script=sci_arttext\&pid=S164698952015000300003

Novack, J; Cañas, A. The Theory Underlying Concept Maps and How to Construct Them, Technical Report IHMC CmapTools, Florida Institute for Human and Machine Cognition. (accesso: 10 de mayo del 2017). Recuperado de http://cmap.ihmc.us/Publications/

Shubauer-Leoni, M. (1986). El desarrollo cognitivo de los niños en la escuela primaria. La psicología del aprendizaje en las diferentes Situaciones pedagógicas. Revista de Educación, 279 (1),pp. 103-106. Recuperado de https://www.mecd.gob.es/revista-de-educacion/ numeros-revista-educacion/numeros-anteriores/1986/re279.html

Argentina. UNESCO, Ministerio de Educación de la Nación. (2012). La evolución biológica actualidad y debates. pp. 22-24. Recuperado dehttps://www.yumpu.com/es/document/view/48864546/la-

Van Lieshout, C. (1986). El aprendizaje y la enseñanza: Una parte del desarrollo. Revista de Educación, 279(1), pp. 122-124. Recuperado de https://www.mecd.gob.es/revista-de-educacion/numeros-revistaeducacion/numeros-anteriores/1986/re279.html

Warnik, B. (2014). Fairness in Teaching Evolution in Public Schools. Philosophical Studies in Education, 45(1), p. 56. Recuperado de https:/ /eric.ed.gov/?id=EJ1043629 